\title{
Modelos para estimativa de variáveis florestais com a utilização de imagens multiespectrais
}

\author{
Igor Eloi Silva Machado*, Micael Moreira Santos², Marcos Giongo, Edmar Vinicius de Carvalho', Eduardo Ganassoli Neto \\ ${ }^{1}$ Universidade Federal do Tocantins, Rua Badejós, Chácaras 69 e 72, Lt. 07, CEP 77402-970, Gurupi, TO, Brasil \\ 2Universidade Federal do Paraná, Av. Pref Lothario Meissner, 3400, CEP 80210-170 , Curitiba, PR, Brasil
}

"Autor correspondente:

igeloi@hotmail.com

Termos para indexação:

Landsat

Inventários florestais

Estimativa de volume

Index terms:

Landsat

Forest inventory

Volume estimation

Histórico do artigo:

Recebido em 09/11/2016

Aprovado em 25/05/2017

Publicado em 30/06/2017

\begin{abstract}
Resumo - Técnicas de sensoriamento remoto em monitoramento vegetacional vêm sendo cada vez mais utilizadas e melhoradas. A utilização dessas técnicas é uma alternativa potencial para embasar inventários florestais. O presente trabalho tem por objetivo estimar variáveis florestais utilizando imagens multiespectrais associadas a informações obtidas em levantamento de campo. Foi estudada uma área de floresta tropical com aproximadamente 44.728,5 ha. Foi feito um inventário florestal $100 \%$, fornecendo a volumetria das árvores com circunferência a 1,30 m acima do solo (CAP) superior a $110 \mathrm{~cm}$. Foi utilizada a cena 227/069 do Landsat 7, sendo feita correção radiométrica da imagem, obtendo-se os valores de reflectância. Foram avaliados três tamanhos de parcelas circulares (40, 80 e $120 \mathrm{~m}$ de raio) para ajuste dos modelos lineares. O comportamento espectral da área apresentou respostas baixas na região do visível (TM1, TM2 e TM3), aumentando no infravermelho próximo (TM4). A banda TM4 apresentou maior correlação com o CAP (R: - 0,5203$)$. O melhor modelo para estimativa do volume exibiu $\mathrm{R}_{\mathrm{aj}}^{2}=0,387 \mathrm{e} \mathrm{S}_{\mathrm{yx}}=30,199 \%$, estimando um volume médio de $39,61 \mathrm{~m}^{3} \mathrm{ha}^{-1}$. Os resultados demonstraram viabilidade do uso de imagens de satélites para estimativa de variáveis dendrométricas.
\end{abstract}

\section{Models of forest variables estimation using multispectral images}

\begin{abstract}
Remote sensing techniques for vegetation monitoring has been more used and improved. These techniques are good alternative to be used as as basis for forest inventories. The present study aims to estimate forest variables using multispectral images associated with data from field survey. The studied area was a tropical rain forest of approximately $44,728.5 \mathrm{ha}$. A forest inventory $100 \%$ was carried out providing the volume of tree species with circumference at $1.30 \mathrm{~m}$ above soil level $(\mathrm{CBH})$ higher than $110 \mathrm{~cm}$. The used satellite was Landsat 7, scene 227/069. A radiometric correction was performed to obtain the reflectance values. Three plots sizes $(40,80,120 \mathrm{~m}$ radius) were assessed for linear models adjustment. The area spectral behave presented low response on visible region (TM1, TM2 and TM3), increasing on near-infrared (TM4). TM4 band presented higher correlation with $\mathrm{CBH}(\mathrm{R}:-0.5203)$. The best model to estimate showed $\mathrm{a} \mathrm{R}_{\text {aj }}{ }_{\mathrm{a}}=0.387$ and $\mathrm{S}_{\mathrm{yx}}=30.199 \%$, estimating an average volume of $39,61 \mathrm{~m}^{3} \mathrm{ha}^{-1}$. The results showed viability to use satellite images to evaluate dendrometric variables.
\end{abstract}




\section{Introdução}

O sensoriamento remoto tem como um dos seus objetivos estudar os fenômenos e processos que acontecem na superfície da Terra, pela análise das interações entre a radiação eletromagnética e os componentes da superfície do planeta em suas mais diversas manifestações (Novo, 2008).

Atualmente, existe um grande número de sensores imageadores que observam a Terra, em comprimentos de onda do visível até micro-ondas, em resoluções espaciais que variam de metros a quilômetros e frequências temporais variando de minutos a semanas ou meses (Rosenqvist et al., 2003). Pela facilidade de aquisição das imagens e confiabilidade dos resultados, os sensores da plataforma Landsat tornaram-se os mais utilizados na área do sensoriamento remoto.

A determinação de variáveis dendrométricas, como volume de madeira, área basal, diâmetro, altura, etc., pode ser trabalhoso e demorado, o que leva ao aumento considerável dos custos, principalmente nas áreas florestais de grande extensão e que estão localizadas em área distantes e pouco acessíveis (Alves et al., 2013).

Para a determinação dos atributos florestais com o subsídio de imagens de satélite é essencial o processamento digital das imagens, que visa melhorar a resolução e realce, facilitando a extração dos dados para associação das informações digitais de um sistema remoto de detecção com características biofísicas e variáveis que são obtidas em campo.

Defries (2008) destaca a importância dos dados adquiridos por meio de sensores ópticos a bordo de satélites, que são de considerável importância para o monitoramento e análise da vegetação. No entanto, sempre é necessário que haja a associação dos dados obtidos de imagens de satélite com dados reais, como os de inventários florestais.

A correlação dessas duas técnicas, sensoriamento remoto e coleta de dados em campo, pode ser uma ótima alternativa para se diminuir os trabalhos com inventários florestais. Variáveis obtidas pela aplicação de modelos estatísticos ou coletadas e medidas em campo, como altura, volume e diâmetro podem apresentar correlação com valores de reflectância obtidos na imagem (Alves et al., 2013).

Ponzoni \& Shimabukuro (2007) indicam que os índices de vegetação são importantes para a caracterização das estruturas florestais. No entanto, a grande maioria dos estudos que envolvem correlação entre elementos espectrais e as variáveis dendrométricas não avaliam o efeito do tamanho das parcelas de amostragem (Reis et al., 2015).

Diante do exposto, o objetivo do presente trabalho foi gerar modelos capazes de estimar a circunferência média a 1,30 $\mathrm{m}$ acima do solo (CAP) e o volume de madeira de uma área florestal com a utilização de imagens multiespectrais, avaliando parcelas circulares, em três dimensões de raio.

\section{Material e métodos}

\section{Área de estudo}

O trabalho foi realizado em um fragmento de floresta tropical, localizado no município de Nova Maringá,

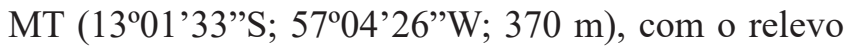
variando de plano a suavemente ondulado, em uma área de 44.728,5 ha (dividida em 3 talhões). A região apresenta clima do tipo tropical com estação seca (Aw) de acordo a classificação climática de Köppen.

No local, foi feito inventário florestal 100\%, como requisito para a elaboração do plano de manejo florestal sustentável, fornecendo a localização espacial e a volumetria de todas as espécies passíveis de corte. Nesse inventário foram medidas a altura comercial $(\mathrm{Hc})$ e a circunferência a $1,30 \mathrm{~m}$ acima do solo (CAP) para todas as árvores com CAP $>110 \mathrm{~cm}$ (Tabela 1).

Foram consideradas como variáveis dendrométricas de interesse para a realização do trabalho o CAP médio $(\mathrm{cm})$ e o volume $\left(\mathrm{m}^{3}\right)$ estimado para cada árvore. Para isto foi utlizado o modelo proposto por modelo de Spurr (Equação 1, $\mathrm{R}^{2}=0,96$ ), conforme Queiroz (1984).

$$
\mathrm{V}=0,077476+0,517897 *\left(\mathrm{DAP}^{2} * \mathrm{Hc}\right)
$$

Sendo: $\mathrm{v}=$ volume, $\mathrm{DAP}=$ diâmetro a $1,30 \mathrm{~m}$ acima do solo, $\mathrm{Hc}=$ altura comercial.

Tabela 1. Análise descritiva das variáveis florestais, por árvore, mensuradas no inventario florestal a $100 \%$, Nova Maringá, MT.

\begin{tabular}{lcccc}
\hline \multicolumn{1}{c}{ Variáveis } & Máximo & Mínimo & Média & $\begin{array}{c}\text { Desvio } \\
\text { padrão }\end{array}$ \\
\hline CAP $(\mathrm{cm})$ & 815 & 110 & 197,807 & 65,958 \\
Altura comercial $(\mathrm{m})$ & 40 & 4 & 9,799 & 1,535 \\
Volume $\left(\mathrm{m}^{3}\right)$ & 40,420 & 0,070 & 2,512 & 2,121 \\
\hline
\end{tabular}

CAP: Circunferência a 1,30 $\mathrm{m}$ acima do solo. 


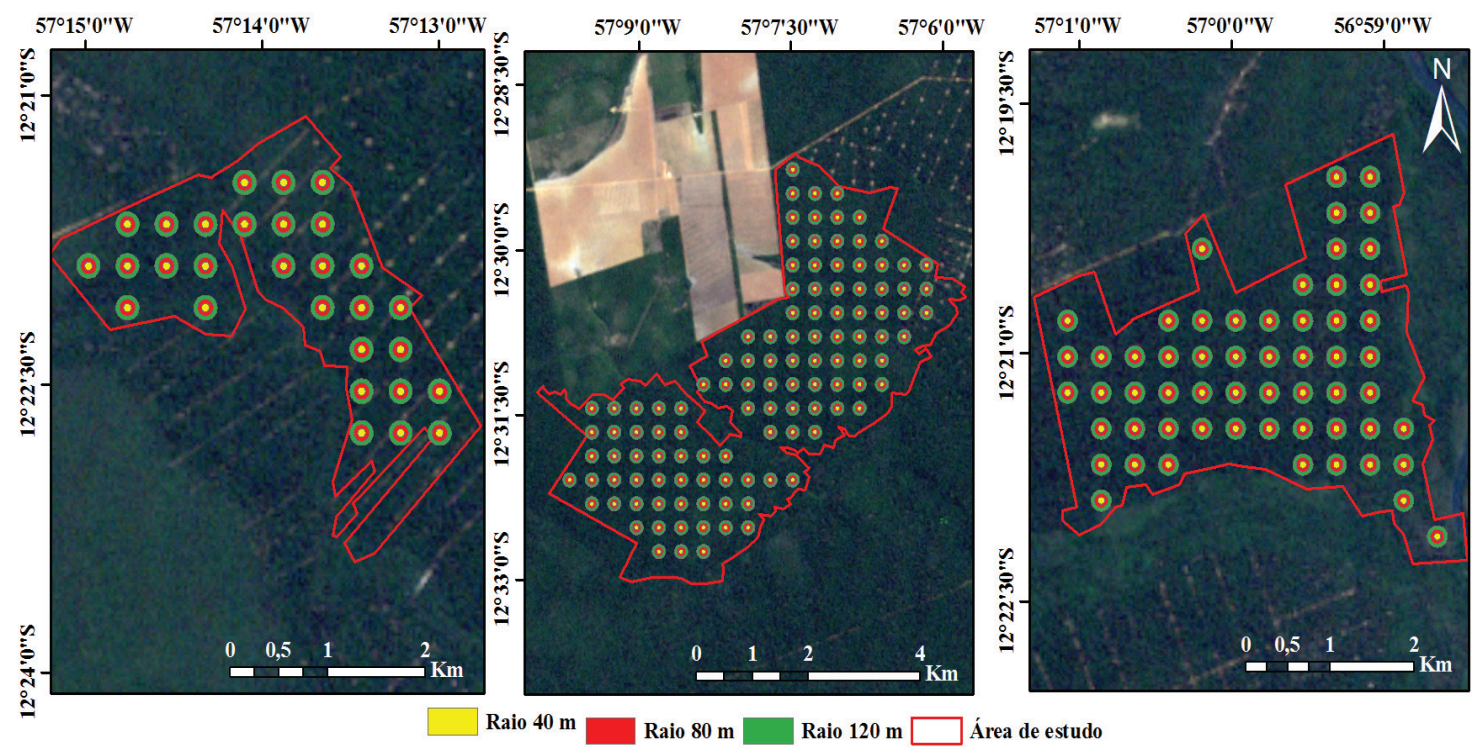

Figura 1. Talhões da área de estudo e os diferentes raios (40, 80 e $120 \mathrm{~m})$ das parcelas amostradas, Nova Maringá, MT, 2016.

\section{Aquisição e processamento da imagem multiespectral}

Na aquisição da imagem, no caso, do satélite Landsat, o primeiro passo foi analisar a disponibilidade de imagens no período em que foi realizado o inventário florestal, que foi de 2000 a 2002. A imagem escolhida foi da órbita/ponto 227/069 do dia 30 de julho de 2001, disponível no site do U. S. Geological Survey (United States, 2016).

A imagem passou por processo de correção atmosférica e radiométrica, visando à redução das degradações nas imagens. Neste processo, foram gerados produtos de reflectância com suas respectivas bandas TM1 (azul: 0,45 a $0,52 \mu \mathrm{m}$ ), TM2 (verde: 0,53 a $0,62 \mu \mathrm{m}$ ), TM3 (vermelho: 0,63 a $0,70 \mu \mathrm{m}$ ) e TM4 (infravermelho: $0,71$ a $1,3 \mu \mathrm{m})$. Na obtenção dos valores de reflectância dos alvos presentes na cena, foi necessário efetuar a transformação de número digital (ND) das bandas em reflectância, dados estes utilizados nas análises de correlação entre a imagem e os dados de campo.

\section{Reflectância}

Nos estudos de sensoriamento remoto encontramse fórmulas genéricas, aplicáveis a diversos sensores, localidades, alvos e períodos, para executar a transformação de ND em reflectância, obtendo-se ao final, na maioria das vezes, uma regressão linear simples para esta conversão. Para a estimativa dos valores de reflectância foi usada a metodologia apresentada por Gürtler et al. (2005), que ajusta o ND de um pixel a um modelo linear, por meio de parâmetros constantes, para obter o valor em reflectância correspondente (Equação 2).

$$
\operatorname{Ref}=\mathrm{i}+(\mathrm{j} \times \mathrm{ND})
$$

Sendo: Ref $=$ valor da reflectância; $\mathrm{ND}=$ número digital; i e j = parâmetros constantes determinados para a cena específica.

O ND varia dentro da imagem de pixel para pixel e as variáveis constantes são: ângulo de elevação solar no momento da obtenção da imagem (dado da imagem), ângulo zenital solar, dia do ano contado consecutivamente a partir de 1 de janeiro até a data da imagem (dia juliano) e distância terra-sol em unidades astronômicas.

As informações de reflectância podem ser extraídas das imagens a partir de diferentes tamanhos de parcelas amostrais dos pixels, onde o pixel central de cada parcela corresponde ao centroide da unidade amostral (Almeida et al., 2014).

\section{Relação imagem espectral e variáveis dendrométricas}

Após as correções da imagem, deu-se início ao processamento dos dados da imagem Landsat 7, sendo 
extraídas as informações das bandas da imagem. Para a determinação dos dados das bandas da imagem foi utilizada amostragem sistemática, considerando intervalo de $400 \mathrm{~m}$ entre os pontos centrais das parcelas, o que gerou um total de 167 parcelas nos três talhões. As parcelas amostradas tiveram 40, 80 e $120 \mathrm{~m}$ de raio.

Com isso, os valores de reflectância das bandas foram gerados dentro dessas parcelas, com obtenção dos valores médios de cada uma. Além das análises de buffer da imagem, também foram extraídos os índices de vegetação (IV): índice de vegetação da diferença normalizada (NDVI), índice de vegetação ajustado do solo (SAVI) e razão simples (SR), obtidos pelas equações 3 a 5, respectivamente (Leal et al., 2013).

$$
\begin{aligned}
& \mathrm{NDVI}=(\mathrm{TM} 4-\mathrm{TM} 3) /(\mathrm{TM} 4+\mathrm{TM} 3) \\
& S A V I=\frac{(T M 4-T M 3)}{(T M 4+T M 3+L)} *(1+L) \\
& S R=T M 4 / T M 3
\end{aligned}
$$

Em que, NDVI = índice de vegetação da diferença normalizada; SAVI = índice de vegetação ajustado do solo; $\mathrm{SR}$ = razão simples; TM3 = reflectância do vermelho; TM4 = reflectância do infravermelho próximo; e L = fator de correção do solo (valor adotado $=0,5$ ).

Além dos valores de TM1, TM2, TM3, TM4 e dos índices de vegetação referentes a cada parcela, foram realizadas transformações ou combinações de variáveis, buscando um melhor ajuste dos futuros modelos para estimativa de variáveis dendrométricas.

Nas análises estatísticas de regressão foram calculadas as correlações entre as variáveis dendrométricas com as respostas espectrais da imagem. Por fim, os modelos foram ajustados (equação 6), aplicando-se a metodologia de Backward para se determinar as variáveis de interesse para os modelos, onde as respostas espectrais (bandas e índices de vegetação) entraram como variáveis independentes e os dados oriundos do inventário florestal (CAP e volume) como variáveis dependentes.

$$
\mathrm{Y}=\beta 0+\beta 1 \mathrm{X} 1+\beta 2 \mathrm{X} 2+\beta 3 \mathrm{X} 3+\beta 4 \mathrm{X} 4+\ldots+\beta \mathrm{nXn}+\varepsilon
$$

Em que, $\mathrm{Y}=$ variável dependente; $\beta \mathrm{n}=$ parâmetros ajustados; $\mathrm{Xn}=$ variáveis de interesse.

Ao final, foram selecionados os três melhores modelos para cada tamanho de parcela, avaliando-se as seguintes estatísticas: coeficiente de determinação ajustado $\left(\mathrm{R}^{2}{ }_{\mathrm{aj}}\right)$, erro padrão da estimativa percentual $\left(\mathrm{S}_{\mathrm{yx}} \%\right.$ ) (equações 7 e 8 , respectivamente) e análise gráfica de resíduo.

$$
R_{a j}^{2}=\left\{1-\left[\left(1-R^{2}\right) *\left(\frac{n-1}{n-p}\right)\right]\right\}
$$

Onde, $\mathrm{R}^{2}=$ coeficiente de determinação; $\mathrm{n}=$ número de observações e $\mathrm{p}=$ número de variáveis independentes.

$$
S_{y x} \%=\left(\frac{S_{y x}}{\bar{Y}}\right) * 100
$$

Onde, $\mathrm{S}_{\mathrm{yx}}=$ erro padrão absoluto e $\overline{\mathrm{Y}}=$ média da variável dependente.

O melhor modelo para estimar volume de acordo com a análise estatística foi utilizado para espacialização do volume, para demonstrar a variação desta variável em toda área estudada, sendo comparado com o mapa de volume real, obtido a partir do inventario $100 \%$.

\section{Resultados}

Os valores médios de reflectância da imagem nos diferentes tipos de parcelas para as bandas TM1, TM2, TM3, TM4 e para os índices avaliados são apresentados na Tabela 2. Pode-se observar que o comportamento espectral apresenta respostas baixas na região do visível $(0,0172$ a 0,0331$)$, aumentando no infravermelho próximo $(0,2946)$.

Tabela 2. Valores médios de reflectância para as diferentes bandas espectrais e índices de vegetação nas parcelas analisadas, na região de Nova Maringá, MT.

\begin{tabular}{ccccccccc}
\hline PARC (RAIO) & $\hat{\text { TM1 }}$ & $\hat{\text { TM2 }}$ & $\hat{\text { TM3 }}$ & $\hat{\text { TM4 }}$ & $\hat{\text { NDVI }}$ & SAVI & SR \\
\hline $40 \mathrm{~m}$ & 0,0172 & 0,0331 & 0,0229 & 0,2946 & 0,8555 & 0,4981 & 13,1466 \\
$80 \mathrm{~m}$ & 0,0173 & 0,0331 & 0,0228 & 0,2945 & 0,8560 & 0,4982 & 13,1684 \\
$120 \mathrm{~m}$ & 0,0173 & 0,0330 & 0,0228 & 0,2943 & 0,8559 & 0,4979 & 13,1720 \\
\hline
\end{tabular}

PARC $($ RAIO) = parcelas com raios de 40, 80 e 120 m; TM1, TM2, TM3 e TM4 = reflectância nas bandas do azul, verde, vermelho e infravermelho próximo, respectivamente; $\mathrm{NDVI}=$ índice de vegetação de diferença normalizada; $\mathrm{SAVI}=$ índice de vegetação de ajuste do solo; $\mathrm{SR}=$ razão simples . 
Na Tabela 3, são apresentados os coeficientes de correlação das variáveis independentes e dependentes. $\mathrm{O}$ maior valor de correlação está entre a TM4 com variáveis florestais na parcela de $120 \mathrm{~m}$ de raio, com $-0,5203$ $(\mathrm{p}<0,05)$ e $-0,4587(\mathrm{p}<0,05)$ para CAP e volume, respectivamente, ou seja, a diminuição da quantidade de energia refletida está associada com aumento do volume, sendo inversamente proporcional.

$\mathrm{Na}$ Tabela 4 estão apresentados os modelos encontrados para estimar CAP com as devidas variáveis independentes e combinações das mesmas e seus parâmetros estatísticos. Pode-se notar a presença da variável independente banda do infravermelho próximo (TM4) em todos os modelos, independentemente do tamanho da parcela.

Tabela 3. Correlação de Pearson das bandas do visível, infravermelho próximo e índices de vegetação com as variáveis dendrométricas CAP $(\mathrm{cm})$ e volume $\left(\mathrm{m}^{3} \mathrm{ha}^{-1}\right)$, na região de Nova Maringá, MT.

\begin{tabular}{|c|c|c|c|}
\hline Atributos & Parcela 40* & Parcela 80* & Parcela 120* \\
\hline CAP X TM1 & 0,1625 & 0,2476 & 0,2907 \\
\hline CAP X TM2 & $-0,0488$ & $-0,2307$ & $-0,3117$ \\
\hline CAP X TM3 & 0,2751 & 0,2614 & 0,2733 \\
\hline CAP X TM4 & $-0,2449$ & $-0,4208$ & $-0,5203$ \\
\hline CAP X NDVI & $-0,2967$ & $-0,3675$ & $-0,4154$ \\
\hline CAP X SAVI & $-0,2791$ & $-0,4293$ & $-0,5149$ \\
\hline CAP X SR & $-0,3300$ & $-0,4074$ & $-0,4652$ \\
\hline VOL X TM1 & 0,1337 & 0,1644 & 0,2068 \\
\hline VOL X TM2 & $-0,1522$ & $-0,3059$ & $-0,3513$ \\
\hline VOL X TM3 & 0,0547 & 0,0923 & 0,1482 \\
\hline VOL X TM4 & $-0,1921$ & $-0,3397$ & $-0,4587$ \\
\hline VOL X NDVI & $-0,1058$ & $-0,2069$ & $-0,2965$ \\
\hline VOL X SAVI & $-0,1771$ & $-0,3175$ & $-0,4322$ \\
\hline VOL X SR & $-0,1595$ & $-0,2450$ & $-0,3497$ \\
\hline \multicolumn{4}{|c|}{$\begin{array}{l}\text { *: Todos os coeficientes foram significativos }(\mathrm{p}<0,05) \text {, pelo teste } \mathrm{t} \text {. CAP } \\
=\text { circunferência a } 1,30 \mathrm{~m} \text { acima do solo }(\mathrm{cm}) ; \mathrm{VOL}=\text { volume de madeira } \\
\text { em } \mathrm{m}^{3} \text {; TM1, TM2, TM3 e TM4 = reflectância nas bandas do azul, verde, } \\
\text { vermelho e infravermelho próximo, respectivamente; NDVI = índice de } \\
\text { vegetação de diferença normalizada; SAVI = índice de vegetação de ajuste } \\
\text { do solo; SR = razão simples. }\end{array}$} \\
\hline
\end{tabular}

Os modelos apresentaram melhores ajustes a medida que os raios das parcelas foram aumentando. Dentre todas as parcelas, o melhor ajuste foi do modelo MC3120 da parcela com raio de $120 \mathrm{~m}$, apresentando $\mathrm{R}_{\text {aj }}^{2}=$ 0,458 e $\mathrm{S}_{\mathrm{yx}} \%=8,062$.

Para o melhor modelo ajustado para a estimativa de CAP $(\mathrm{cm})$, fez-se a análise de resíduo em percentual (Figura 2), apresentando uma variação de $-20 \%$ a $20 \%$.

Na Tabela 5 são apresentados os modelos ajustados para a estimativa de volume com suas devidas variáveis selecionadas, de acordo as combinações realizadas, e seus parâmetros estatísticos. É possível notar, como nos ajustes para CAP, que a variável TM4 se encontra como variável independente na maioria dos modelos para estimar volume, o que demonstra que a mesma pode ser considerada variável de grande interesse para trabalhos com objetivos semelhantes a este estudo. As outras variáveis constantes nos modelos para determinação do volume foram os índices de vegetação (NDVI, SAVI e $\mathrm{SR})$.

O melhor ajuste foi do modelo MV2-120, da parcela com raio de $120 \mathrm{~m}$, que apresentou $\mathrm{R}_{\text {aj }}^{2}=0,387$ e $\mathrm{S}_{\mathrm{yx}} \%$ $=30,199$ (Tabela 5).

Para o melhor modelo ajustado para a estimativa de volume $\left(\mathrm{m}^{3} \mathrm{ha}^{-1}\right)$, fez-se a análise de resíduo em percentual (Figura 3), apresentando uma variação de $-110 \%$ a $50 \%$.

A partir da distribuição de resíduos do modelo MV2-120 (Figura 3), observa-se superestimação do volume em áreas com menor volume.

Aplicando-se o modelo MV2-120, foi gerada imagem da área com a espacialização do volume atual de madeira, de acordo com valores de reflectância do pixel. Os valores dos pixels presentes na imagem representam o valor de volume $\left(\mathrm{m}^{3} \mathrm{ha}^{-1}\right)$, podendo ser comparado com o mapa de volume gerado a partir do inventário 100\% (Figura 4).

$\mathrm{O}$ volume médio de madeira determinado com a aplicação do modelo MV2-120 foi de $39,61 \mathrm{~m}^{3} \mathrm{ha}^{-1}$, apresentando na área total $(44.728,45 \mathrm{ha})$ volume de $1.771 .693,90 \mathrm{~m}^{3}$. O volume médio de madeira determinado a partir do inventario $100 \%$ para árvores com CAP $>110 \mathrm{~cm}$ foi de $38,35 \mathrm{~m}^{3} \mathrm{ha}^{-1}$, com volume total de $1.715 .336,06 \mathrm{~m}^{3}$. 
Tabela 4. Modelos lineares ajustados para estimativa da circunferência a 1,30 m do solo em floresta tropical, na região de Nova Maringá, MT, e seus parâmetros estatísticos.

\begin{tabular}{|c|c|c|c|c|}
\hline $\begin{array}{l}\text { Raio das } \\
\text { parcelas }\end{array}$ & $\begin{array}{c}\text { Códigos dos } \\
\text { modelos }\end{array}$ & Modelos & $R^{2} a j$ & Syx\% \\
\hline \multirow{3}{*}{$40 \mathrm{~m}$} & MC1-40 & $\begin{array}{l}\widehat{\mathrm{CA}}=-13095,4114+(20902,9979 \mathrm{NDVI})+(-11106,1374 \mathrm{SAVI})+(91,3321 \mathrm{SR})+ \\
\left(42670071,8031 \mathrm{TM} 1^{3}\right)+\left(-4998998,3991 \mathrm{TM} 2^{3}\right)+\left(21233164,3144 \mathrm{TM} 3^{3}\right)+(2,5553 \\
(1 / \mathrm{TM} 1))+(-50,2946(1 / \mathrm{TM} 3))+(99497,4737(\mathrm{TM} 4 * \mathrm{TM} 2))+(-39546451,0177 \\
(\mathrm{TM} 1 * \mathrm{TM} 2 * \mathrm{TM} 3))+(13714,9736(1 / \mathrm{SR}))\end{array}$ & 0,1670 & 15,0950 \\
\hline & MC2-40 & $\begin{array}{l}\widehat{\mathrm{CAP}}=-12677,5666+(20059,0783 \mathrm{NDVI})+(-9615,4668 \mathrm{SAVI})+(88,5722 \mathrm{SR})+ \\
\left(48671699,1947 \mathrm{TM}^{3}\right)+\left(23323556,9336 \mathrm{TM} 3^{3}\right)+(1,9592(1 / \mathrm{TM} 1))+(-50,7315 \\
(1 / \mathrm{TM} 3))+(56038,9581(\mathrm{TM} 4 * \mathrm{TM} 2))+(-49091535,4668(\mathrm{TM} 1 * \mathrm{TM} 2 * \mathrm{TM} 3))+ \\
(13216,6974(1 / \mathrm{SR}))\end{array}$ & 0,1700 & 15,0690 \\
\hline & MC3-40 & $\begin{array}{l}\widehat{\mathrm{CAP}}=-12306,6250+(19722,4429 \mathrm{NDVI})+(-9664,9732 \mathrm{SAVI})+(87,3914 \mathrm{SR}) \\
+\left(46851272,2187 \mathrm{TM} 1^{3}\right)+\left(25150306,2115 \mathrm{TM} 3^{3}\right)+(-49,4739(1 / \mathrm{TM} 3))+ \\
(64279,2796(\mathrm{TM} 4 * \mathrm{TM} 2))+\left(-55167580,7525\left(\mathrm{TM} 1^{*} \mathrm{TM} 2 * \mathrm{TM} 3\right)\right)+(13253,2224 \\
(1 / \mathrm{SR}))\end{array}$ & 0,1720 & 15,0510 \\
\hline \multirow{3}{*}{$80 \mathrm{~m}$} & MC1-80 & $\begin{array}{l}\widehat{\mathrm{CA}} \mathrm{P}=2693,8783+(-3935,8835 \mathrm{NDVI})+(-4649,8746 \mathrm{SAVI})+\left(63707417,5682 \mathrm{TM}^{3}\right) \\
+\left(-12971407,4536 \mathrm{TM} 2^{3}\right)+\left(6682753,2717 \mathrm{TM}^{3}\right)+\left(-17458,0926 \mathrm{TM} 4^{3}\right)+(40,4084 \\
(1 / \mathrm{TM} 2))+(349576,8205(\mathrm{TM} 4 * \mathrm{TM} 2))+\left(-71476218,8149\left(\mathrm{TM} 1^{*} \mathrm{TM} 2 * \mathrm{TM} 3\right)\right)\end{array}$ & 0,3350 & 9,6290 \\
\hline & MC2-80 & $\begin{array}{l}\widehat{\mathrm{CA}}=3273,7300+(-4568,4198 \mathrm{NDVI})+(-4151,6190 \mathrm{SAVI})+(51573861,1058 \\
\left.\mathrm{TM} 1^{3}\right)+\left(-13452686,2417 \mathrm{TM} 2^{3}\right)+\left(-15518,0731 \mathrm{TM} 4^{3}\right)+(36,6124(1 / \mathrm{TM} 2))+ \\
(323990,9177(\mathrm{TM} 4 * \mathrm{TM} 2))+\left(-57054524,3403\left(\mathrm{TM} 1^{*} \mathrm{TM} 2 * \mathrm{TM} 3\right)\right)\end{array}$ & 0,3360 & 9,6170 \\
\hline & MC3-80 & $\begin{array}{l}\widehat{\mathrm{CA}}=4815,3959+(-7655,0227 \mathrm{NDVI})+\left(62798724,0216 \mathrm{TM}^{3}\right)+(-8251056,6466 \\
\left.\mathrm{TM} 2^{3}\right)+\left(-21126,3851 \mathrm{TM} 4^{3}\right)+(28,4894(1 / \mathrm{TM} 2))+(257259,3612(\mathrm{TM} 4 * \mathrm{TM} 2)) \\
+\left(-70466441,1319\left(\mathrm{TM} 1^{*} \mathrm{TM} 2 * \mathrm{TM} 3\right)\right)\end{array}$ & 0,3340 & 9,6830 \\
\hline \multirow{3}{*}{$120 \mathrm{~m}$} & MC1-120 & $\begin{array}{l}\widehat{\mathrm{CAP}}=-2664,6437+(123,1544 \mathrm{SR})+\left(21327281,4151 \mathrm{TM} 1^{3}\right)+(-19029459,2730 \\
\left.\mathrm{TM} 2^{3}\right)+\left(-18733,7512 \mathrm{TM} 4^{3}\right)+\left(-0,1071 \mathrm{SR}^{3}\right)+(-4,1004(1 / \mathrm{TM} 1))+(51,4214 \\
(1 / \mathrm{TM} 2))+(401489,7232(\mathrm{TM} 4 * \mathrm{TM} 2))+(-22493,4015(\mathrm{TM} 4 * \mathrm{SAVI})+ \\
(-39027674,8362(\mathrm{TM} 1 * \mathrm{TM} 2 * \mathrm{TM} 3))+(14847,2388(1 / \mathrm{SR}))\end{array}$ & 0,4560 & 8,0770 \\
\hline & MC2-120 & $\begin{array}{l}\widehat{\mathrm{CA}}=-2346,5699+(91,0295 \mathrm{SR})+\left(36247741,1276 \mathrm{TM}^{3}\right)+(-21984855,1392 \\
\left.\mathrm{TM} 2^{3}\right)+\left(-21133,8674 \mathrm{TM} 4^{3}\right)+\left(-0,0763 \mathrm{SR}^{3}\right)+(55,1539(1 / \mathrm{TM} 2))+(446558,7106 \\
(\mathrm{TM} 4 * \mathrm{TM} 2))+(-25272,0783(\mathrm{TM} 4 * \mathrm{SAVI})+(-39204450,5630(\mathrm{TM} 1 * \mathrm{TM} 2 * \mathrm{TM} 3)) \\
+(11519,2699(1 / \mathrm{SR}))\end{array}$ & 0,4570 & 8,0670 \\
\hline & MC3-120 & $\begin{array}{l}\widehat{\mathrm{CAP}}=-1688,7764+(25,9525 \mathrm{SR})+\left(34754345,8010 \mathrm{TM} 1^{3}\right)+(-20957288,2499 \\
\left.\mathrm{TM} 2^{3}\right)+\left(-23160,7592 \mathrm{TM} 4^{3}\right)+(57,5161(1 / \mathrm{TM} 2))+(439694,8863(\mathrm{TM} 4 * \mathrm{TM} 2))+ \\
(-23025,6341(\mathrm{TM} 4 * \mathrm{SAVI})+(-37465998,8983(\mathrm{TM} 1 * \mathrm{TM} 2 * \mathrm{TM} 3))+(7421,6828(1 / \\
\mathrm{SR}))\end{array}$ & 0,4580 & 8,0620 \\
\hline
\end{tabular}

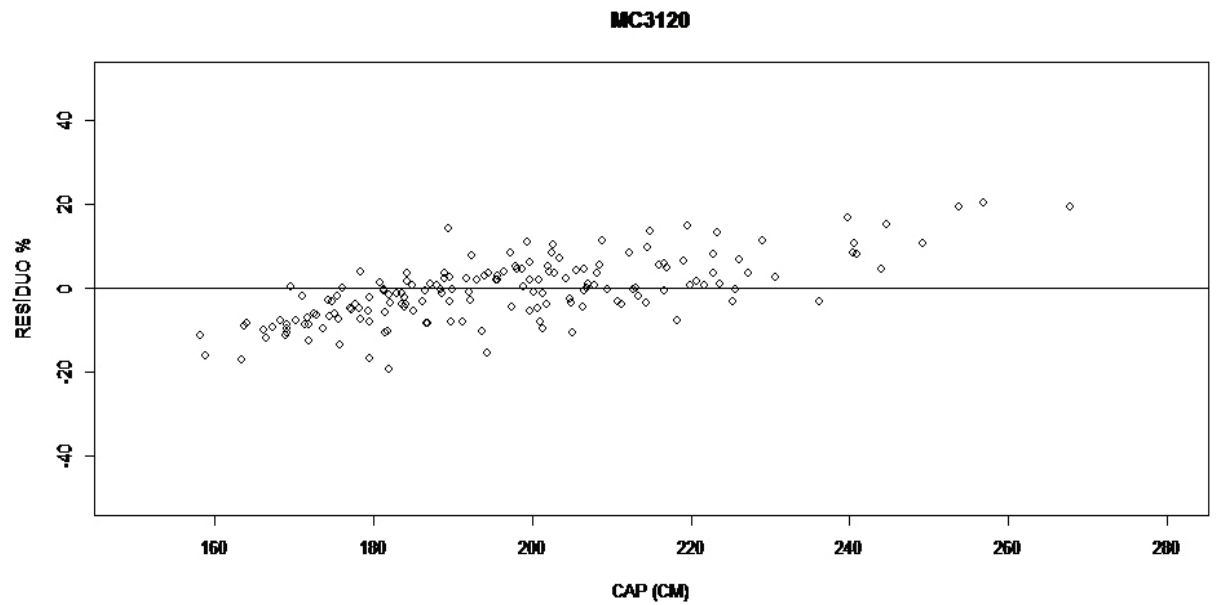

Figura 2. Análise de resíduos (\%) do melhor modelo ajustado para estimativa de CAP (cm), MC3-120, em Nova Maringá, MT. 
Tabela 5. Modelos lineares encontrados para estimativa de volume $\left(\mathrm{m}^{3}\right)$ em floresta tropical, na região de Nova Maringá, MT, e seus parâmetros estatísticos.

\begin{tabular}{|c|c|c|c|c|}
\hline $\begin{array}{l}\text { Raios das } \\
\text { parcelas }\end{array}$ & $\begin{array}{l}\text { Códigos dos } \\
\text { modelos }\end{array}$ & Modelos & $R^{2} a j$ & Syx\% \\
\hline \multirow{3}{*}{$40 \mathrm{~m}$} & MV1-40 & $\begin{array}{l}\checkmark=-11443,5231+(12442,9984 \mathrm{NDVI})+(-7109,1199 \mathrm{SAVI}) \\
+(134,5310 \mathrm{SR})+\left(10004501,6118 \mathrm{TM} 1^{3}\right)+(-13640192,7894 \\
\left.\mathrm{TM}^{3}\right)+\left(-2573106,9522 \mathrm{TM}^{3}\right)+\left(-22823,1218 \mathrm{TM} 4^{3}\right)+ \\
\left(-0,0582 \mathrm{SR}^{3}\right)+(2,0318(1 / \mathrm{TM} 1))+(38,5347(1 / \mathrm{TM} 2)) \\
+(-32,0850(1 / \mathrm{TM} 3))+(264998,0259(\mathrm{TM} 4 * \mathrm{TM} 2))+ \\
(17609,1093(1 / \mathrm{SR}))\end{array}$ & 0,1280 & 54,9970 \\
\hline & MV2-40 & $\begin{array}{l}\hat{V}=-12138,3804+(13880,1669 \mathrm{NDVI})+(-7870,0421 \mathrm{SAVI}) \\
+(131,1368 \mathrm{SR})+\left(9957522,5005 \mathrm{TM} 1^{3}\right)+(-13222077,8233 \\
\left.\mathrm{TM} 2^{3}\right)+\left(-20492,0610 \mathrm{TM}^{3}\right)+\left(-0,0557 \mathrm{SR}^{3}\right)+(2,0313 \\
(1 / \mathrm{TM} 1))+(36,8462(1 / \mathrm{TM} 2))+(-33,2769(1 / \mathrm{TM} 3))+ \\
(255155,7743(\mathrm{TM} 4 * \mathrm{TM} 2))+(17301,4177(1 / \mathrm{SR}))\end{array}$ & 0,1330 & 54,8380 \\
\hline & MV3-40 & $\begin{array}{l}\hat{V}=-11797,9511+(13510,5383 \mathrm{NDVI})+(-7531,8989 \mathrm{SAVI}) \\
+(130,0540 \mathrm{SR})+\left(2757916,5100 \mathrm{TM} 1^{3}\right)+(-12707908,5058 \\
\left.\mathrm{TM} 2^{3}\right)+\left(-20122,7478 \mathrm{TM} 4^{3}\right)+\left(-0,0561 \mathrm{SR}^{3}\right)+(35,9750(1 / \\
\mathrm{TM} 2))+(-32,0665(1 / \mathrm{TM} 3))+(246844,4969(\mathrm{TM} 4 * \mathrm{TM} 2)) \\
+(17360,3416(1 / \mathrm{SR}))\end{array}$ & 0,1300 & 54,9110 \\
\hline \multirow{3}{*}{$80 \mathrm{~m}$} & MV1-80 & $\begin{array}{l}\vee=-1767,7164+(1729,2486 \mathrm{NDVI})+(45,0182 \mathrm{SR})+ \\
\left(-7915134,5504 \mathrm{TM}^{3}\right)+\left(-17215,8989 \mathrm{TM} 4{ }^{3}\right)+(-5,8650(1 / \\
\mathrm{TM} 1))+(-12,0823(1 / \mathrm{TM} 3))+(103271,8112(\mathrm{TM} 4 * \mathrm{TM} 2)) \\
+(-21601977,0587(\mathrm{TM} 1 * \mathrm{TM} 2 * \mathrm{TM} 3))+(7999,1265(1 / \mathrm{SR}))\end{array}$ & 0,2610 & 37,8070 \\
\hline & MV2-80 & $\begin{array}{l}\widehat{V}=-265,4688+(30,4128 \mathrm{SR})+\left(-8708833,3133 \mathrm{TM} 2^{3}\right)+ \\
\left(-15303,2645 \mathrm{TM} 4{ }^{3}\right)+(-5,9443(1 / \mathrm{TM} 1))+(-6,5449(1 / \\
\mathrm{TM} 3))+(112905,4170(\mathrm{TM} 4 * \mathrm{TM} 2))+(-21841164,7869 \\
(\mathrm{TM} 1 * \mathrm{TM} 2 * \mathrm{TM} 3))+(5645,0860(1 / \mathrm{SR}))\end{array}$ & 0,2640 & 37,7250 \\
\hline & MV3-80 & $\begin{array}{l}\widehat{V}=-511,0162+(10,2979 \mathrm{SR})+\left(-11597976,9003 \mathrm{TM} 2^{3}\right)+ \\
\left(-15747,8743 \mathrm{TM} 4^{3}\right)+(-3,6026(1 / \mathrm{TM} 1))+(131436,0973 \\
(\mathrm{TM} 4 * \mathrm{TM} 2))+(-11440117,0015(\mathrm{TM} 1 * \mathrm{TM} 2 * \mathrm{TM} 3))+ \\
(4154,0807(1 / \mathrm{SR}))\end{array}$ & 0,2580 & 37,8690 \\
\hline \multirow{3}{*}{$120 \mathrm{~m}$} & MV1-120 & $\begin{array}{l}\widehat{V}=96,6044+\left(36317895,1169 \mathrm{TM}^{3}\right)+(26260534,2190 \\
\left.\mathrm{TM}^{3}\right)+\left(-3321,8333 \mathrm{TM}^{3}\right)+\left(3231,4982 \mathrm{NDVI}^{3}\right)+(-5,8869 \\
(1 / \mathrm{TM} 1))+(-10,9728(1 / \mathrm{TM} 3))+(69657,5774(\mathrm{TM} 4 * \mathrm{TM} 2)) \\
+(-11841,2660(\mathrm{TM} 4 * \mathrm{SAVI}))+(-65099649,1278 \\
(\mathrm{TM} 1 * \mathrm{TM} 2 * \mathrm{TM} 3))+(3065,8100(1 / \mathrm{SR}))\end{array}$ & 0,3840 & 30,2670 \\
\hline & MV2-120 & $\begin{array}{l}\widehat{v}=315,0732+\left(33009965,5955 \mathrm{TM}^{3}\right)+(26332495,0171 \\
\mathrm{TM} 33)+\left(3881,6718 \mathrm{NDVI}^{3}\right)+(-5,9993(1 / \mathrm{TM} 1))+(-13,0677 \\
(1 / \mathrm{TM} 3)+(65007,4636(\mathrm{TM} 4 * \mathrm{TM} 2))+(-15786,1690 \\
(\mathrm{TM} 4 * \mathrm{SAVI}))+(-61541977,2120(\mathrm{TM} 1 * \mathrm{TM} 2 * \mathrm{TM} 3))+ \\
(2830,2185(1 / \mathrm{SR}))\end{array}$ & 0,3870 & 30,1990 \\
\hline & MV3-120 & $\begin{array}{l}\forall=124,0353+\left(18406970,4073 \mathrm{TM}^{3}\right)+(3134,9741 \\
\left.\mathrm{NDVI}^{3}\right)+(-9,0083(1 / \mathrm{TM} 1))+(-7,9583(1 / \mathrm{TM} 3))+ \\
(29894,8791(\mathrm{TM} 4 * \mathrm{TM} 2))+(-10132,4021(\mathrm{TM} 4 * \mathrm{SAVI}))+ \\
(-35506087,0828(\mathrm{TM} 1 * \mathrm{TM} 2 * \mathrm{TM} 3))+(3437,5475(1 / \mathrm{SR}))\end{array}$ & 0,3820 & 30,3100 \\
\hline
\end{tabular}




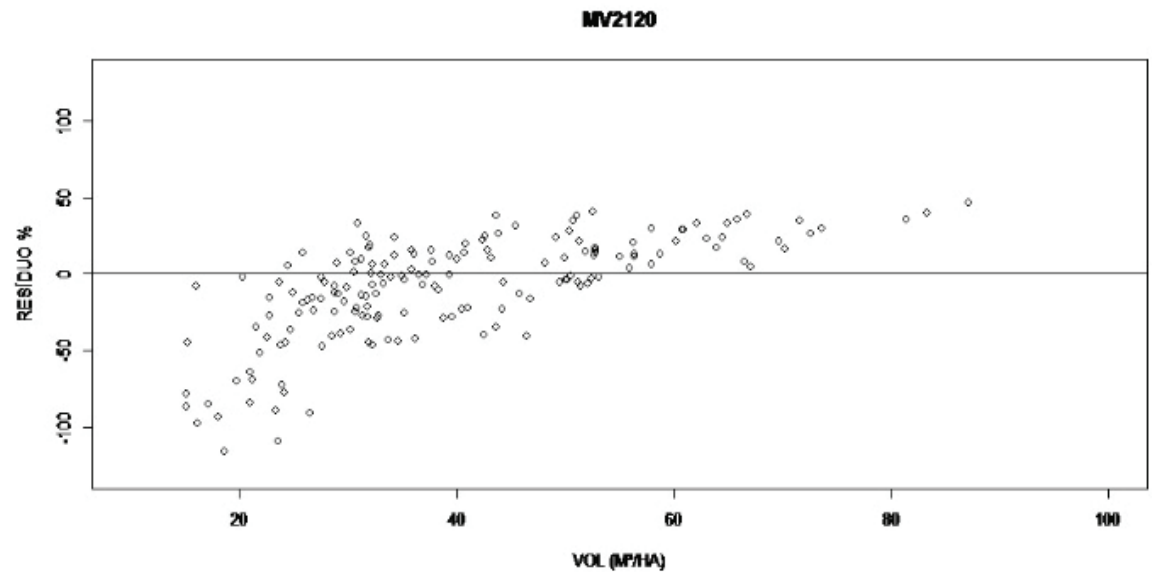

Figura 3. Análise gráfica de resíduo (\%) do melhor modelo para estimativa de volume $\left(\mathrm{m}^{3} \mathrm{ha}^{-1}\right), \mathrm{MV} 2-120$, em Nova Maringá, MT.

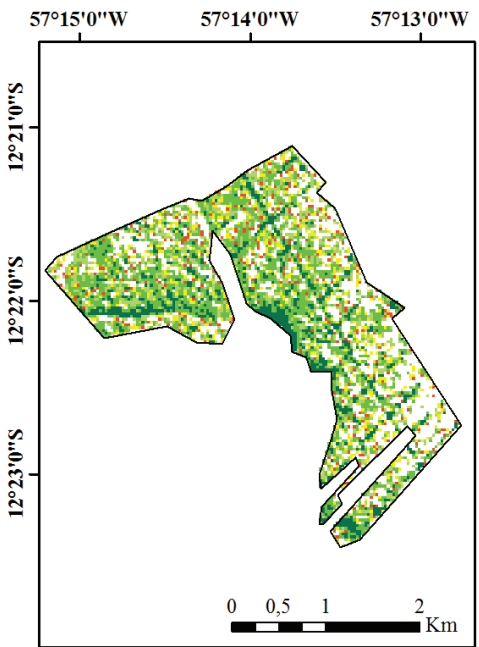

$\square 0 \mathrm{~m}^{3} / \mathrm{ha} \quad 1,0-20,0 \mathrm{~m}^{3} / \mathrm{h}$

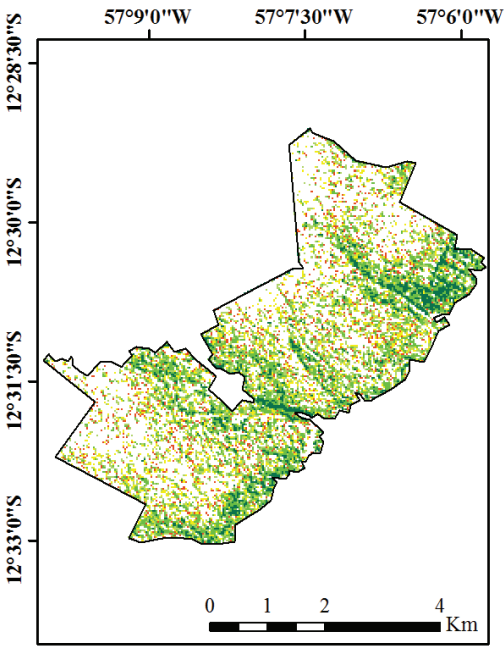

$20,0-60,0 \mathrm{~m}^{3} / \mathrm{ha} \square 60,0-120,0 \mathrm{~m}^{3} / \mathrm{ha}$

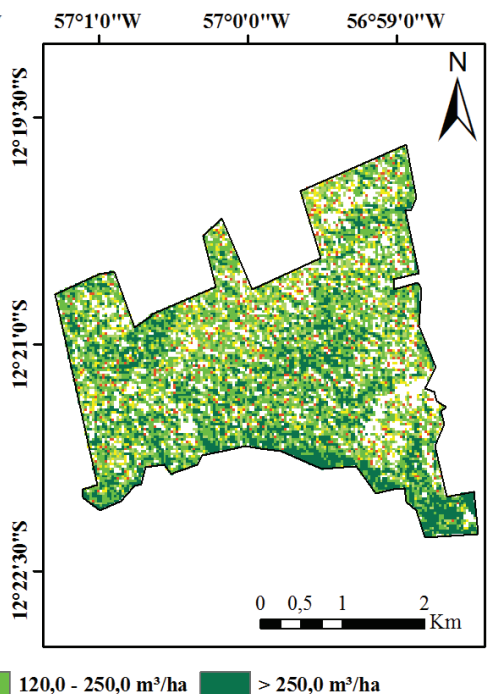

$120,0-250,0 \mathrm{~m}^{3} / \mathrm{ha}$

$250,0 \mathrm{~m}^{3} / \mathrm{ha}$
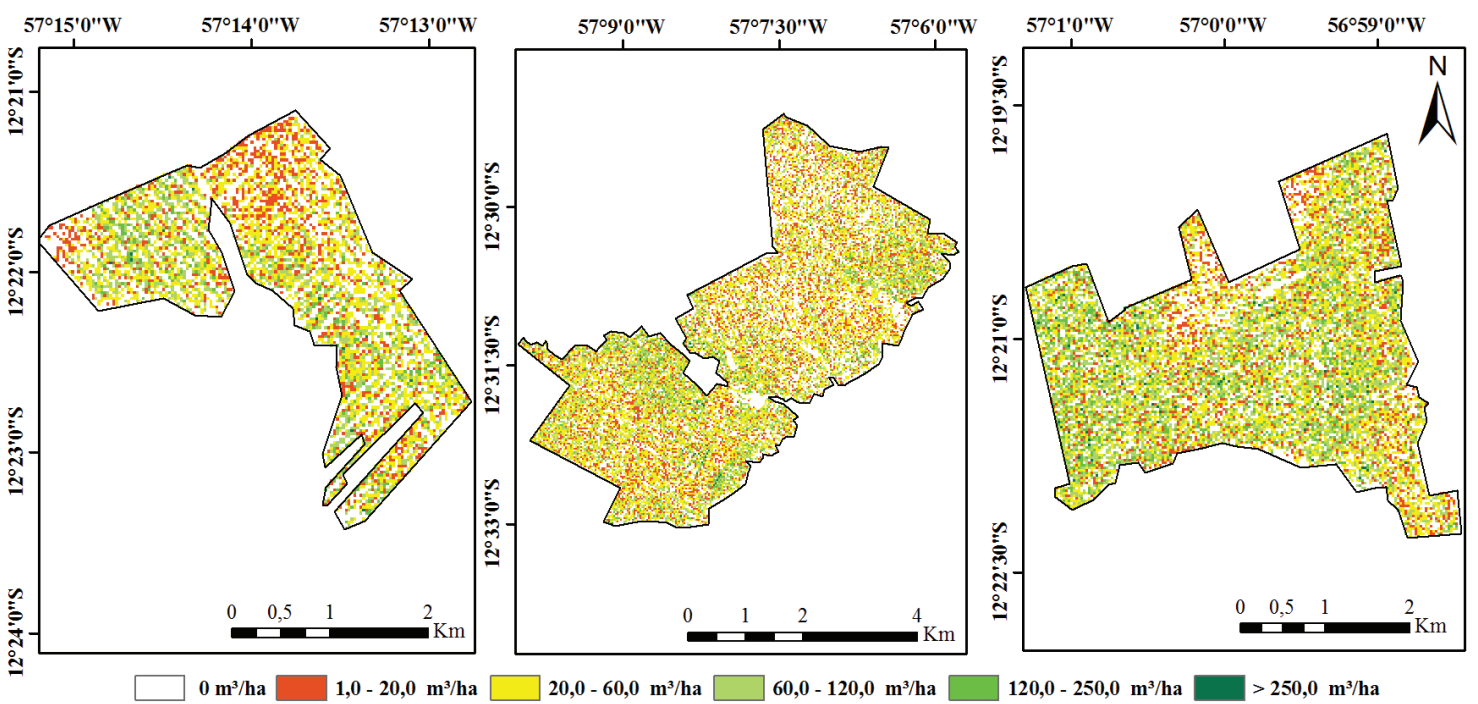

Figura 4. Espacialização do volume $\left(\mathrm{m}^{3} \mathrm{ha}^{-1}\right)$ estimado pelo modelo MV2-120 (a), e mapa de volume $\left(\mathrm{m}^{3} \mathrm{ha}^{-1}\right)$ encontrado no inventário $100 \%(\mathrm{~b})$.

Pesq. flor. bras., Colombo, v. 37, n. 90, p. 171-181, abr.jun. 2017 


\section{Discussão}

\section{Valores médios de reflectância}

Jensen (2009) observou uma diminuição de absorção na região espectral da faixa do verde $(0,53 \mu \mathrm{m}$ a $0,62 \mu \mathrm{m})$. Resultado que pode ser observado neste trabalho, onde os maiores valores médios de reflectância nas parcelas foram obtidos no espectro do visível (infravermelho próximo), na região do verde, devido a menor absorção. Watzlawick et al. (2009) concluíram que nos comprimentos de onda do infravermelho próximo $(0,71 \mu \mathrm{m}$ a $1,3 \mu \mathrm{m})$ estão os maiores valores de reflectância da vegetação, devido às estruturas celulares das folhas, com influência direta da quantidade de água presente.

Os índices de vegetação de diferença normalizada (NDVI) e de ajuste do solo (SAVI) expressam valores que representam a densidade da vegetação. Quanto mais próximos de 1, mais densa é a floresta avaliada (Rêgo et al., 2012). Os valores de SAVI e NDVI variaram de 0,4979 a 0,4982 e 0,8555 a 0,8560 , respectivamente, refletindo características de floresta densa. Os valores de SAVI foram menores, devido ao fator de correção da interferência do solo.

\section{Correlação entre as variáveis dendrométricas e digitais}

É possível observar que a medida que a área das parcelas aumenta, ocorre aumento da correlação de todas as bandas (TM1, TM2, TM3 e TM4) e índices (NDVI, SAVI e SR) com as variáveis dendrométricas (CAP e volume). Essa resposta é esperada, pois quanto maior o raio da parcela, mais pixels serão analisados para gerar os valores de reflectâncias.

As maiores correlações (Tabela 4) aconteceram entre a banda do infravermelho próximo (TM4) com as variáveis florestais. Lu et al. (2004) avaliaram as relações das variáveis florestais com imagens de TM/Landsat na Bacia Amazônia Brasileira, onde encontraram correlação inversa entre TM4 e DAP $(-0,6180)$, resultado semelhante ao deste trabalho quando correlacionado com o CAP $(-0,4208)$.

Berra et al. (2012) estudaram a correlação entre volume de madeira de plantios de Eucalyptus sp. com dados espectrais obtidos em imagens TM/Landsat $5 \mathrm{e}$ encontraram baixa correlação entre volume e a banda do vermelho (TM3). Isso se deve à máxima absorção da radiação neste comprimento de onda pela clorofila. Esses autores observaram também correlação positiva entre o volume de madeira e a banda TM4 $(0,1700)$ e altos valores de correlação com os índices NDVI $(0,7900)$ e SR $(0,8200)$. Esses resultados diferem dos resultados obtidos nesse trabalho, possivelmente pelas diferenças de respostas espectrais próprias de um plantio homogêneo comparado a uma área de floresta nativa. Em florestas nativas o crescimento e a conformação das copas são irregulares e desuniformes, como isso as grandes copas interferem na reflectâncias das árvores menores e logicamente nos valores de correlações.

Pode-se perceber que a maioria das correlações entre as variáveis dendrométricas e as respostas espectrais foram negativas. Pinheiro et al. (2009), estimando biomassa aérea em áreas de Cerrado utilizando imagens Landsat, encontraram valores de correlação negativos na faixa do visível do espectro eletromagnético e positivos para o infravermelho próximo. Isso pode estar relacionado com a grande quantidade de sombras no dossel produzidas pelos indivíduos adultos (Canavesi \& Ponzoni, 2007).

Estes resultados evidenciam que o tamanho e estrutura das copas afetam os valores de correlações entre dados espectrais e características dendrométricas. Segundo Li et al. (2014), a estrutura do dossel, a área foliar que ocupa as copas e a reflexão do fundo do dossel são fatores condicionantes das mudanças da reflectância das florestas nas imagens de sensoriamento remoto, sendo características específicas de cada fitofisionomia (ecossistema) em cada região de ocorrência.

\section{Análise dos modelos ajustados para CAP}

A maior importância da TM4 como variável independente atestada por maiores valores de correlações observados nesse trabalho é corroborada pelos resultados de Canavesi \& Ponzoni (2007) que avaliaram as relações entre variáveis dendrométricas de plantios de Eucalyptus sp. e valores de reflectância de imagens Landsat. Esses autores observaram que ao realizarem a análise de regressão, somente os valores provenientes da TM4 foram considerados como variável independente.

Vale a pena destacar que os índices de vegetação (NDVI, SAVI e SR) podem ser usados como variáveis independentes para os ajustes de modelos para estimativa de CAP, apresentando participação significativa em todos os tamanhos de parcela. 
Os valores de $\mathrm{R}_{\text {aj }}^{2}$ para os modelos de CAP variaram de 0,167 a 0,458 , mostrando que quando se aumenta o raio da parcela, obtém-se melhor resposta das variáveis independentes (espectrais) junto a variável florestal. Thenkabail et al. (2003) encontraram $\mathrm{R}^{2}=0,52 \mathrm{com}$ modelos apresentando a banda do infravermelho médio $(1,55 \mu \mathrm{m}$ a $1,75 \mu \mathrm{m})$ de imagens Landsat 7 como variável independente.

\section{Análise dos modelos ajustados para volume}

A presença constante dos índices de vegetação (NDVI, SAVI e SR) nos modelos para estimativa de volume (Tabela 5), é um resultado semelhante ao obtido por Berra et al. (2012) na estimativa de volume de madeira de plantios de Eucalyptus sp., onde as melhores equações foram ajustadas com pelo menos um dos índices de vegetação (NDVI ou SR).

Canavesi et al. (2010) avaliaram estimativa de volume de madeira em plantios de Eucalyptus spp. utilizando dados hiperespectrais e dados topográficos e encontraram valores de $\mathrm{R}_{\text {aj }}^{2}$ de 0,5890 a 0,7040 , resultados superiores aos observados nesse trabalho, possivelmente por se tratar de uma área de floresta plantada, mais homogênea.

A aparência da cobertura vegetal de uma imagem de satélite é produto da interação de diversos parâmetros e fatores ambientais, que influenciam na resposta espectral de cada fisionomia e, com isso, nos estudos de associação. Portanto, os modelos selecionados no presente estudo podem ser utilizados para florestas tropicais com características semelhantes, mas os resultados podem variar em outros tipos de fisionomia.

Ainda, Lu et al. (2004) afirmam que as características vegetacionais de cada ecossistema influenciam a resposta espectral do mesmo, afetando diretamente nos resultados dos modelos ajustados para determinação de variáveis dendrométricas estimadas através de imagens de satélites.

\section{Conclusões}

O uso de imagens de satélites é viável para o ajuste de modelos para estimativa de variáveis dendrométricas.

Os índices de vegetação de diferença normalizada e de ajuste do solo e razão simples apresentam grande importância na geração de modelos lineares para a estimativa da circunferência a $1,30 \mathrm{~m}$ acima do solo (CAP) e do volume em florestas tropicais.
A correlação entre as variáveis digitais e dendrométricas aumentou de acordo com o aumento do raio das parcelas, exibindo melhor correlação entre CAP e reflectância na banda infravermelho próximo. As parcelas com $120 \mathrm{~m}$ de raio apresentaram melhores ajustes de modelos para CAP $(\mathrm{cm})$ e volume $\left(\mathrm{m}^{3} \mathrm{ha}^{-1}\right)$.

\section{Referências}

Almeida, A. Q. et al. Relações empíricas entre características dendrométricas da Caatinga brasileira e dados TM Landsat 5. Pesquisa Agropecuária Brasileira, v. 49, n. 4, p. 306-315, 2014. DOI: $10.1590 / \mathrm{S} 0100-204 X 2014000400009$.

Alves, M. V. G. et al. Aplicação de k-nearest neighbor em imagens multispectrais para a estimativa de parâmetros florestais. Floresta, v. 43, n. 3, p. 351-362, 2013. DOI: 10.5380/rf.v43i3.18083.

Berra, E. F. et al. Estimativa do volume total de madeira em espécies de eucalipto a partir de imagens de satélite Landsat. Ciência Florestal, v. 22, n. 4, p. 853-864, 2012. DOI: 10.5902/198050987566.

Canavesi, V. et al. Estimativa de volume de madeira em plantios de Eucalyptus spp. utilizando dados hiperespectrais e dados topográficos. Revista Árvore, v. 34, n. 3, p. 539-549, 2010. DOI: 10.1590/S0100-67622010000300018.

Canavesi, V. \& Ponzoni, F. J. Relações entre variáveis dendrométricas de plantios de Eucalyptus sp. e valores de FRB de superfície de imagens do sensor TM/Landsat 5. In: SIMPÓSIO BRASILEIRO DE SENSORIAMENTO REMOTO, 13., 2007, Florianópolis. Anais... São José dos Campos: INPE, 2007. p. 1619-1625.

Defries, R. Terrestrial vegetation in the coupled human-earth system: contributions of remote sensing Annu. Review of Environment and Resources, v. 33, n. 1, p. 369-390, 2008. DOI: 10.1146/annurev. environ.33.020107.113339.

Gürtler, S. et al. Planilha eletrônica para o cálculo da reflectância em imagens TM e ETM+ LANDSAT. Revista Brasileira de Cartografia, v. 57, n. 2, p. 162-167, 2005.

Jensen, J. R. Sensoriamento remoto do ambiente: uma perspectiva em recursos terrestres. São José dos Campos: Parêntese, 2009. 598 p.

Leal, F. A. et al. Índice de vegetação na estimativa do volume em um povoamento de eucalipto. Enciclopédia Biosfera, v. 9, n. 17, p. $1638,2013$.

Li, D. et al. Estimating the age of deciduous forests in northeast China with Enhanced Thematic Mapper Plus data acquired in different phenological seasons. Journal of Applied Remote Sensing, v. 8, n. 1, p. 083670: 1-20, 2014. DOI: 10.1117/1.JRS.8.083670.

$\mathrm{Lu}, \mathrm{D}$. et al. Relationships between forest stand parameters and Landsat TM spectral responses in the Brazilian Amazon Basin. Forest Ecology and Management, v. 198, n. 1-3, p. 149-167, 2004. DOI: 10.1016/j.foreco.2004.03.048.

Novo, E. M. L. de M. Sensoriamento remoto: princípios e aplicações. 3. ed. São Paulo, SP: Blucher, 2008. 387 p. 
Pinheiro, E. da S. et al. Imagens Landsat e QuickBird são capazes de gerar estimativas precisas de biomassa aérea de Cerrado? In: SIMPÓSIO BRASILEIRO DE SENSORIAMENTO REMOTO, 14., 2009, Natal. Anais... São José dos Campos: INPE, 2009. p. 2913-2920.

Ponzoni, F. J. \& Shimabukuro, Y. E. Sensoriamento remoto no estudo da vegetação. São José dos Campos, SP: INPE, 2007. 150 p.

Queiroz, W. T. Análise de fatores pelo método da máxima verossimilhança: aplicação ao estudo da estrutura de florestas tropicais. 1984. 109 f. Tese (Doutorado em Engenharia Florestal) Universidade de São Paulo, Piracicaba.

Rêgo, S. C. A. et al. Análise comparativa dos índices de vegetação NDVI e SAVI no município de São Domingos do Cariri-PB. Revista Geonorte, v. 2, n. 4, p. 1217-1229, 2012.

Reis, A. A et al. Amostragens em imagens de sensoriamento remoto para a determinação da correlação entre características dendrométricas e dados espectrais. In: SIMPÓSIO BRASILEIRO DE SENSORIAMENTO REMOTO, 17, 2015, João Pessoa. Anais... São José dos Campos: INPE. 2015. p. 4699-4704.
Rosenqvist, A. et al. A review of remote sensing technology in support of the Kyoto Protocol. Environmental Science \& Policy, v. 6, n. 5, p. 441-455, 2003. DOI: 10.1016/S1462-9011(03)00070-4.

Thenkabail, P. S. Detecting floristic structure and pattern across topographic and moisture gradients in a mixed species Central African forest using IKONOS and Landsat-7 ETM+ images. International Journal of Applied Earth Observation and Geoinformation, v. 4, n. 3, p. 255-270, 2003. DOI: 10.1016/S0303-2434(03)00006-0.

United States. Department of the Interior. U. S. Geological Survey. Earth explorer. Washington, DC, [2016]. Disponível em: <http:// earthexplorer.usgs.gov/> Acesso em: 10 jan. 2016.

Watzlawick, L. F. et al. Estimativa de biomassa e carbono em floresta com araucária utilizando imagens do satélite IKONOS II. Ciência Florestal, v. 19, n. 2, p. 169-181, 2009. DOI: 10.5902/19805098408. 
